INSTITUT NATIONAL DE RECHERCHE EN INFORMATIQUE ET EN AUTOMATIQUE

\title{
Reconciling Distance Functions and Level Sets
}

José Gomes and Olivier Faugeras

$\mathbf{N}^{\circ} 3666$

Avril 1999

THÈME 3

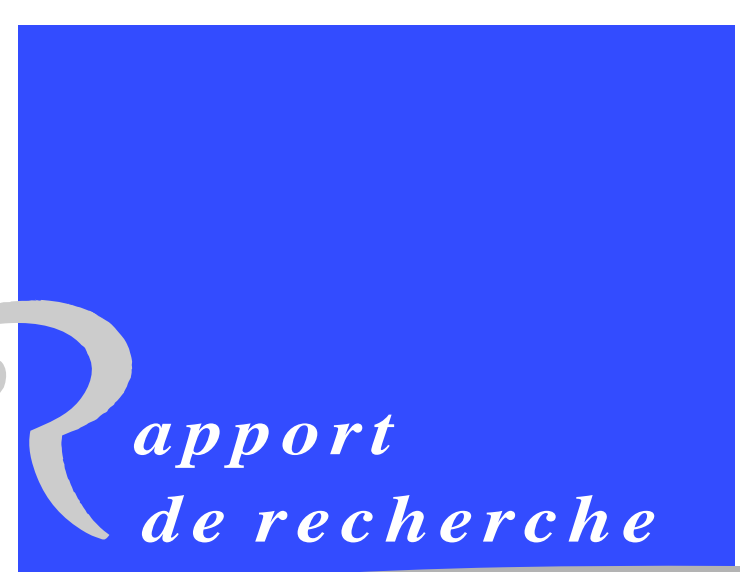





\title{
Reconciling Distance Functions and Level Sets
}

\author{
José Gomes and Olivier Faugeras \\ Thème 3 - Interaction homme-machine, \\ images, données, connaissances \\ Projet Robotvis \\ Rapport de recherche $\mathrm{n}^{\circ} 3666$ - Avril 1999 - 15 pages
}

\begin{abstract}
This paper is concerned with the simulation of the Partial Differential Equation (PDE) driven evolution of a closed surface by means of an implicit representation. In most applications, the natural choice for the implicit representation is the signed distance function to the closed surface. Osher and Sethian propose to evolve the distance function with a Hamilton-Jacobi equation. Unfortunately the solution to this equation is not a distance function. As a consequence, the practical application of the level set method is plagued with such questions as when do we have to "reinitialize" the distance function? How do we "reinitialize" the distance function? Etc... which reveal a disagreement between the theory and its implementation. This paper proposes an alternative to the use of Hamilton-Jacobi equations which eliminates this contradiction: in our method the implicit representation always remains a distance function by construction, and the implementation does not differ from the theory anymore. This is achieved through the introduction of a new equation. Besides its theoretical advantages, the proposed method also has several practical advantages which we demonstrate in three applications: (i) the segmentation of the human cortex surfaces from MRI images using two coupled surfaces [26], (ii) the construction of a hierarchy of Euclidean skeletons of a 3D surface, (iii) the reconstruction of the surface of 3D objects through stereo [12].
\end{abstract}

Key-words: Geodesic Active Contours, Front Propagation, Level Set Methods, Distance Function Preservation, Velocity Extension, Euclidean Skeletons, Cortex Segmentation from MRI, Stereo from Level Sets. 


\section{Réconcilier les Fonctions Distance et les Surfaces de Niveaux}

Résumé : Cet article concerne la résolution des Équations aux Dérivées Partielles (EDP) décrivant la représentation implicite d'une surface fermée en mouvement. Dans la plupart des applications, la représentation implicite naturelle est la distance signée à la surface fermée. Osher et Sethian proposent de soumettre cette fonction implicite à l'action d'une équation de HamiltonJacobi. Malheureusement, aucune fonction distance n'est solution de cette EDP, ce qui est une contradiction. En conséquence, la mise en oeuvre de la méthode dite des "Surfaces de Niveaux", pose la question du rétablissement de la fonction distance: comment le faire ? , quand le faire ? ... Toutes questions auxquelles la théorie proposée ne répond pas.

Par l'introduction d'une nouvelle EDP, cet article propose une alternative à l'utilisation des équations de Hamilton-Jacobi qui élimine cette contradiction. Avec cette nouvelle technique, la fonction implicite est, par construction et à chaque instant, la fonction distance à la surface évoluant, ce qui élimine toute question sur le rétablissement de la fonction distance. Outre ses avantages théoriques, cette approche a des avantages pratiques qui sont démontrés dans le cadre de trois applications: (i) la segmentation du cortex humain à partir de données IRM, (ii) la construction d'une hiérarchie de squelettes euclidiens d'une famille de surfaces, (iii) la reconstruction de scènes tridimentionnelles par stéréovision.

Mots-clés : Contours Actifs Géodésiques, Méthode des Surfaces de Niveaux, Préservation de la Fonction Distance, Squelettes Euclidiens, Segmentation du Cortex à partir d'IRM, Stéréovision 


\section{Contents}

1 Introduction and previous work $\quad 4$

2 Why Hamilton-Jacobi equation (3) does not preserve distance functions. 5

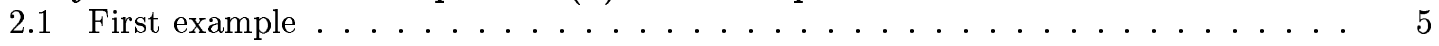

2.2 Second example . . . . . . . . . . . . . . . . . . . 5

3 Why we should preserve the distance function. $\quad 6$

4 How to preserve the signed distance function. $\quad 6$

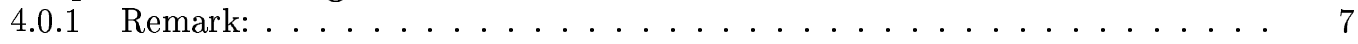

5 Implementation $\quad \mathbf{8}$

5.1 First step: computation of $\beta$ on $\mathcal{Z} \ldots \ldots \ldots \ldots$. . . . . . . . . . . . . . . . . . . . . .

5.2 Second step: computation of $B$ on the narrow band . . . . . . . . . . 8

6 Applications $\quad 9$

6.1 Cortex segmentation using coupled surfaces. . . . . . . . . . . . . . . . . . 9

6.2 Extraction of the skeleton of an evolving surface. . . . . . . . . . . . . . 12

6.3 Stereo reconstruction via level sets. . . . . . . . . . . . . . . . 13

7 Conclusion $r$ 


\section{Introduction and previous work}

We consider a family of hypersurfaces $\mathcal{S}(\mathbf{p}, t)$ in $\mathbb{R}^{3}$, where $\mathbf{p}$ parameterizes the surface and $t$ is the time, that evolve according to the following PDE:

$$
\frac{\partial \mathcal{S}}{\partial t}=\beta \mathcal{N}
$$

with initial conditions $\mathcal{S}(t=0)=\mathcal{S}_{0}$, where $\mathcal{N}$ is the inward unit normal vector of $\mathcal{S}, \beta$ is a velocity function and $\mathcal{S}_{0}$ is some initial closed surface.

Methods of curves evolution for segmentation, tracking and registration were introduced in computer vision by Kass, Witkin and Terzopoulos [15]. These evolutions were reformulated by Caselles, Kimmel and Sapiro [7] and by Kichenassamy et al. [16] in the context of PDE-driven curves and surfaces. There is an extensive literature that addresses the theoretical aspects of these PDE's and offers geometrical interpretations as well as results of uniqueness and existence $[13,14,9]$. Level set methods were first introduced by Osher and Sethian in [21] in the context of fluid mechanics and provide both a nice theoretical framework and efficient practical tools for solving such PDE's. In those methods, the evolution (1) is achieved by means of an implicit representation of the surface $\mathcal{S}$.

The key idea in Osher and Sethian's approach is to introduce the function $u: \mathbb{R}^{3} \times \mathbb{R} \rightarrow \mathbb{R}$ such that

$$
u(\mathcal{S}, t)=0 \quad \forall t
$$

By differentiation (and along with $\mathcal{N}=-\frac{\nabla \mathbf{u}}{|\nabla \mathbf{u}|}$ and (1)), we obtain the Hamilton-Jacobi ${ }^{1}$ equation:

$$
\frac{\partial u}{\partial t}=\beta|\nabla u|
$$

with initial conditions $u(\cdot, 0)=u_{0}($.$) , where u_{0}$ is some initial function $\mathbb{R}^{3} \rightarrow \mathbb{R}$ such that $u_{0}\left(\mathcal{S}_{0}\right)=0$. It has been proved that for a large class of functions $u$ and $u_{0}$, the zero level set at time $t$ of the solution of (3) is the solution at time $t$ of (1).

Regarding the function $u_{0}$, it is most often chosen to be the signed distance function to the closed surface $\mathcal{S}_{0}$. This particular implicit function can be characterized by the two equations:

$$
\left\{x \in \mathbb{R}^{3}, u_{0}(x)=0\right\}=\mathcal{S}_{0} \text { and } \nabla u_{0}=1
$$

Indeed, the magnitude of the gradient of $u_{0}$ is equal to the magnitude of the derivative of the distance function from $\mathcal{S}_{0}$ in the direction normal to $\mathcal{S}_{0}$, i.e., it is equal to 1 .

It is known from [5] that the solution $u$ of (3) is not the signed distance function to the solution $\mathcal{S}$ of (1). This causes several problems which are analyzed in the following section.

It is also important to notice that $\beta$ in (3) is defined in $\mathbb{R}^{3}$ whereas in (1) it is defined on the surface $\mathcal{S}$. The extension of $\beta$ from $\mathcal{S}$ to the whole domain $\mathbb{R}^{3}$ is a crucial point for the analysis and implementation of (3). There are mainly two ways of doing this.

(i) Most of the time this extension is natural. For example, if $\beta=H_{\mathcal{S}}$, the mean curvature of $\mathcal{S}$ in (1), one can choose $\beta=H_{u}$, the mean curvature of the level set of $u$ passing though $x$ in (3).

(ii) In some cases $[24,20,2]$, this extension is not possible. Then one may assign to $\beta(x)$ in (3) the value of $\beta(y)$ in (1) where $y$ is the closest point to $x$ belonging to $\mathcal{S}$. The problem with this extension is that it hides an important dependence of $\beta$ in (3) with respect to $u$ and we show in section 4 that in this case (3) is not a Hamilton-Jacobi equation.

The thrust of this paper is a reformulation of the level set methods introduced by Osher and Sethian in [21] to eliminate some of the problems that are attached to it, e.g. the need to reinitialize periodically the distance function or the need to "invent" a velocity field away from the evolving front or zero level set. The implications of our work are both theoretical and practical.

\footnotetext{
${ }^{1}$ The difference between a Hamilton-Jacobi equation and a general first order PDE is that the unknown function (here $u$ ) does not appear explicitly in the equation.
} 


\section{Why Hamilton-Jacobi equation (3) does not preserve dis- tance functions.}

In this section, we suppose that $\beta$ is extended as explained in (i). The fact that the solutions to Hamilton-Jacobi equations of the form (3) are not distance functions has been proved formally in [5]. A convincing geometrical interpretation of this fact is now given through two short examples.

\section{$2.1 \quad$ First example}

Let us consider the problem of segmenting a known object (an ellipse) in an image by minimizing the energy of a curve [8]. Let us force the initial curve to be exactly the solution (the known ellipse) and initialize $u_{0}$ to the signed distance function to this ellipse, then evolve $u$ with the Hamilton-Jacobi equation (3).

It is obvious that the zero level set of $u$ (let us call $\mathcal{S}_{0}$ this ellipse) will not evolve, since it is the solution to $(1)$ and $\beta\left(x \in \mathcal{S}_{0}\right)=0$.

Notice however that replacing 0 by $\epsilon \in \mathbb{R}$ in (2) implies by differentiation the same equation (3), which means that the $\epsilon$ level set of $u$ (let us call this $\mathcal{S}_{\epsilon}$ curve) also evolves according to $\frac{\partial \mathcal{S}}{\partial t}=\beta \mathcal{N}$. In consequence, $\beta\left(x \in \mathcal{S}_{\epsilon}\right) \neq 0$ and $\mathcal{S}_{\epsilon}$ evolves toward $\mathcal{S}_{0}$ in order to minimize its energy (cf. fig. $(1))$.

time $t=0$

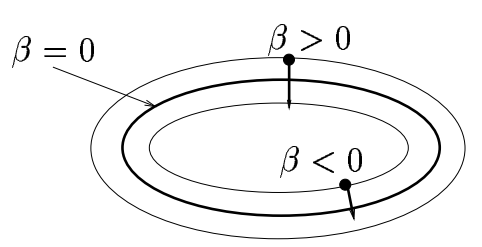

time $t>0$

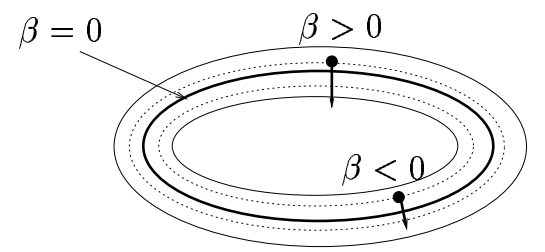

$\mathcal{S}_{0}$

$\mathcal{S}_{ \pm \varepsilon}(0)$

$\mathcal{S}_{ \pm \varepsilon}(t)$

Figure 1: All the level sets of $u$ (shown as single curves) move towards the ellipse $\mathcal{S}_{0}$ in order to minimize their own energy with the effect that the distance function is not preserved.

This shows that the shock wave equation (3) requires that all the level sets of $u$ should converge to the ellipse $\mathcal{S}_{0}$ and therefore that $|\nabla u|$ increases dangerously.

\subsection{Second example}

A point $M$ with coordinate $x \in \mathbb{R}$ and energy $E(x)=\frac{x^{2}}{2}$ is moving along the real line in order to minimize its energy. We force the point $M$ to be at $x_{0} \neq 0$ at $t=0$. The level set version of this problem is to define $u_{0}$ on the real line as $u_{0}(x)=x-x_{0}$ and to evolve $u$ with the Hamilton-Jacobi equation $\frac{\partial u}{\partial t}=x \frac{\partial u}{\partial x}$. The solution is $u(x, t)=e^{t} x-x_{0}$. The figure (2) shows $u$ at 3 time instants $\left(0=t_{0}<t_{1}<t_{2}\right)$. The zero level set of $u$ is indeed traveling to the origin $O$ but the slope of $u$ is $\frac{\partial u}{\partial x}=e^{t}$ and increases exponentially in time.

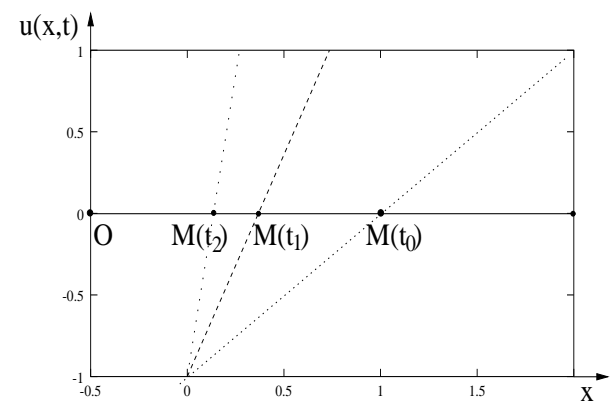

Figure 2: The point $M$ moves on the horizontal line in order to minimize its energy $E(x)=\frac{x^{2}}{2}$. The function $u$, initially of slope 1 , becomes more and more vertical. 
The second example is a rephrasing of what happens in the normal direction to the evolving curve in the first example. It is now obvious why driving all the level sets of $u$ with (3) cannot conserve distance functions and in addition leads to unbounded values of $|\nabla u|$. In practical applications, one is compelled to "reinitialize" the implicit function $u$ to be a distance function which is obviously a contradiction and which shows a gap between the theory and its real application.

In the next section, we convince the reader that maintaining $u$ as a distance function (i.e. such that $|\nabla u|=1$ ) during all the time of the evolution is definitely desirable, sometimes crucial.

\section{Why we should preserve the distance function.}

There are at least two reasons for preserving the signed distance function to the evolving surface, a theoretical one and a practical one.

(i) From the theoretical viewpoint, the parameterized description of $\mathcal{S}$ (seen as a subset of $\mathbb{R}^{3}$ ) and its signed distance function $u$ are equivalent descriptions. Indeed, given any surface $\mathcal{S}$, its signed distance function is uniquely defined. Conversely, any implicit function $u$ satisfying $|\nabla u|=1$ is the signed distance function to a surface plus a constant (this last constant is taken equal to 0 on the surface) [4]. Since these descriptions are equivalent, one can transpose immediately properties of the first one into properties of the second one and vice versa. For example, $\mathbf{u}$ has converged if and only if $\mathcal{S}$ has converged (which is not true with Hamilton-Jacobi equation (3) according to the last section).

Moreover, one can deduce interesting intrinsic properties of $\mathcal{S}$ by a local knowledge of $u$. In [3], it is proved that the second fundamental form of $\mathcal{S}$ can be computed using the derivatives of the squared distance function. In addition, some applications in medical image analysis such as the segmentation of the cortex using two coupled surfaces [26] assume that the distance between the surfaces is known at any time. As a last example, the computation of the skeleton of a surface requires the detection of the singularities of its distance function [18].

(ii) From the practical viewpoint, the numerical approximation of the derivatives of $u$ by finite differences requires the choice of a spatial step $d x$. One chooses a small $d x$ if the slope (the gradient) of the function is large and a larger $d x$ if the function has small variations. Since level sets are most often implemented on regular grids, it is more efficient to use the same step $d x=1$ for each grid point. It is obvious that this approximation is more accurate if the norm of the gradient of $u$ is known which is the case with distance functions since $|\nabla u|=1$. Keeping $|\nabla u|$ bounded assures that the derivatives of $u$ are always computable without the need to "reinitialize" $u$.

We now describe a new approach that preserves the signed distance function and therefore meets these two requirements.

\section{How to preserve the signed distance function.}

In this section, we suppose that $u_{0}=u(., 0)$ is initialized at $t=0$ as the signed distance function to the initial surface $\mathcal{S}_{0}$.

The basic idea is to change equation (3) in such a way that at each time instant $u$ is the signed distance function to the solution $\mathcal{S}$ of (1). In order to achieve this goal, we look for a function $B: \mathbb{R}^{3} \times \mathbb{R}^{+} \rightarrow \mathbb{R}$ such that $\frac{\partial u}{\partial t}=B$ and which satisfies the two constrains: (i) $x \rightarrow u(x,$.$) is a$ distance function, (ii) the zero level set of $u$ evolves according to (1).

We express these constrains with the system of equations:

$$
\left\{\begin{aligned}
B_{\mid u=0} & =\beta \\
\frac{\partial u}{\partial t} & =B \\
|\nabla u| & =1
\end{aligned}\right.
$$

where $B_{\mid u=0}$ denotes the restriction of $B$ to the zero level set of $u$. By differentiating (6) and (7), we obtain:

$$
\nabla\left(\frac{\partial u}{\partial t}\right)=\nabla B \text { and } \frac{\nabla u}{|\nabla u|} \cdot \frac{\partial \nabla u}{\partial t}=0
$$


using the Schwartz equality $\frac{\partial \nabla u}{\partial t}=\nabla\left(\frac{\partial u}{\partial t}\right)$, we get:

$$
\nabla u \cdot \nabla B=0
$$

which, together with (5) and (6) determines the function $B$. Relation (9) states that the function $B$ does not vary along the characteristics of $u$ (the characteristics of $u$ are the integral curves of $\nabla u)$. It also means that the characteristics of $u$ and $B$ are orthogonal.

In order to go one step further in the resolution of the system, we must recall an important property [4]: the characteristics of distance functions are straight lines (cf. fig. (3)).

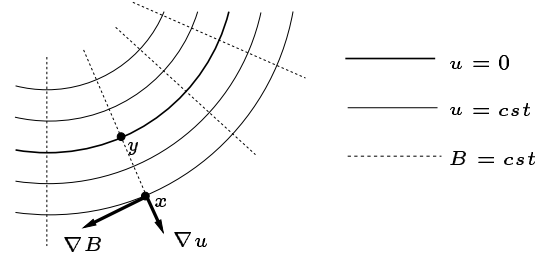

Figure 3: Characteristic curves of the field $\nabla \mathbf{u}$.

This implies that $B$ is constant along straight lines. These lines (or rays) intersect the zero level set of $u$ at a point where $B$ is known according to (5).

Given any point $x \in \mathbb{R}^{3}$, an equation of the characteristic of $u$ passing through $x$ is $\lambda \rightarrow x-\lambda \nabla u$. Since the distance of $x$ to the zero level is $u(x)$ and $|\nabla u(x)|=1$, the point $y=x-u \nabla u$ is on the zero level set of $u$. Notice that $y$ is the closest point to $x$ such that $u(y)=0$. According to the last reasoning, we have $B(x)=B(y)=\beta(x-u \nabla u)$. Therefore, the solution to the initial system is:

$$
\frac{\partial u}{\partial t}=\beta(x-u \nabla u)
$$

with initial condition $u(., 0)=u_{0}($.$) . This equation is the main result of the paper. Note that$ equation (10) is not a Hamilton-Jacobi equation since $u$ appears in the right-hand side and plays a major role. An interpretation of (10) is the following: the zero level set of $u$ is driven by $\frac{\partial u}{\partial t}=\beta$ as proposed by Osher and Sethian. The evolution of this particular surface geometrically defines (by propagation) the evolution of all other level sets.

\subsubsection{Remark:}

a posteriori, one guesses that the integral version of equation (10) is the equation $u(\mathcal{S}+\lambda \mathcal{N})=$ $\lambda \forall t, \lambda$. This can be proved by differentiation with respect to $t$ and $\lambda$. It states that the surface parallel to $\mathcal{S}$ at distance $\lambda$ from $\mathcal{S}$ should be the $\lambda$ level set of $u$. This is to be compared to the constrain $u(\mathcal{S}, t)=0 \quad \forall t$ introduced by Osher and Sethian.

The uniqueness of the closest point $y$ to $x$ such that $u(y)=0$ is only guaranteed if $\nabla u(x)$ exists. The set of points of $\mathbb{R}^{3}$ where $\nabla u$ is not defined is called the skeleton of $\mathcal{S}$ (cf. fig. (4)).

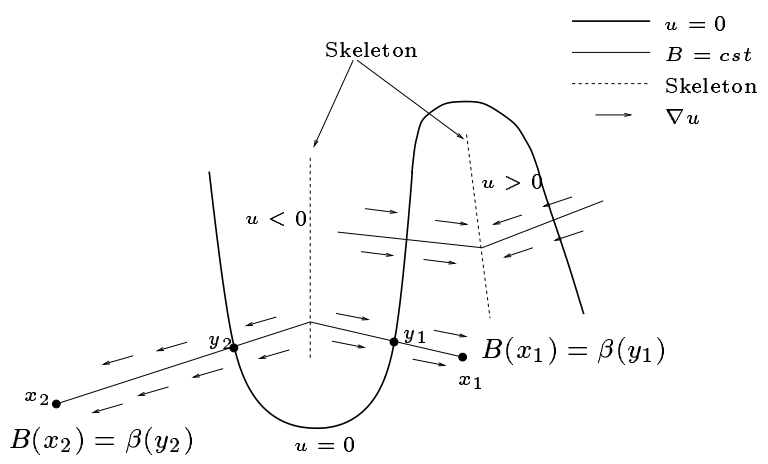

Figure 4: The skeleton of the zero level set is determined by the points where $\nabla u$ is not defined. 
Skeletons are very important in computer vision $[6,17,22]$. Since it turns out that they are a byproduct of our new proposed evolution, we describe in the next section an implementation of equation (10) in which special care is taken of the computation of the skeleton.

\section{Implementation}

In this section, we propose a straightforward implementation of the previous theory. $u$ is initialized as the signed distance function to the initial surface. We fix $u$ at a particular instant $t$ and compute the real field $B(x, t)=\beta(x-u \nabla u)$ on a narrow band $[10,19,1]$ of $\mathcal{S}$. Once $B$ is known, $u$ can be updated by $u(x, t+d t)=u(x, t)+B(x, t) d t$. The computation of $B$ is done in two steps corresponding respectively to equations (5) and (9). The difficulty is that we work on a discrete grid and this can have dramatic consequences if proper care is not taken of the sampling effects.

In order to deal with those effects, we introduce some notations. Points of $\mathbb{R}^{3}$ such that none of their coordinates is an integer will be denoted by lower case letters, e.g. $x$, and called real points. Points of $\mathbb{N}^{3}$, where $\mathbb{N}$ is the set of integers, will be denoted by upper case letters, e.g. $X$, and called voxels. We can think of $x$ as a point falling in a cube formed by eight voxels. We note $V(x)$ this set of eight voxels.

If $f$ is a function defined on $\mathbb{R}^{3}$, and $x$ is a real point such that the values of $f$ are known at all voxels of $V(x)$, we note $f_{l}(x)$ the value of the trilinear interpolation at $x$. In detail, if $x=\left(x_{1}, x_{2}, x_{3}\right)=\left(n_{1}+\epsilon_{1}, n_{2}+\epsilon_{2}, n_{3}+\epsilon_{3}\right)$, where $n_{i} \in \mathbb{N}$ and $0 \leq \epsilon_{i}<1$, then we have by a simple linear interpolation $f_{l}\left(x_{1}, x_{2}, x_{3}\right)=\left(1-\epsilon_{1}\right) f\left(n_{1}, x_{2}, x_{3}\right)+\epsilon_{1} f\left(n_{1}+1, x_{2}, x_{3}\right)$. By applying recursively this rule to $f\left(n_{1}, x_{2}, x_{3}\right)$ and $f\left(n_{1}+1, x_{2}, x_{3}\right)$, one expresses $f_{l}(x)$ as a linear combination of the samples of $f$ at the voxels of $L(x)$, the weights being third order polynomials of the coordinates $\left(\epsilon_{1}, \epsilon_{2}, \epsilon_{3}\right)$.

Let $A(X)$ be the 26-neighborhood of the voxel $X$. Since generically the zero level set of $u$ is composed of real points, we need to determine when a voxel $X$ is adjacent to this zero level set. Consider the function $C_{u}$ defined on the voxels of the grid such that $C_{u}(X)=0$ if $u(X)>0$ and $C_{u}(X)=1$ if $u(X) \leq 0$. A voxel $X$ is said to be adjacent to the zero level set of $u$ if $\exists Y \in A(X), C_{u}(Y) \neq C_{u}(X)$. We call $\mathcal{Z}$ the set of voxels adjacent to the zero level set of $u$. We are now in position to describe the two steps of our computation.

\subsection{First step: computation of $\beta$ on $\mathcal{Z}$}

The first step is the computation of $\beta$ on $\mathcal{Z}$. These values are stored in a temporary buffer called $B^{\mathcal{Z}}$. There are two ways to do this. If $\beta$ is defined on $\mathbb{R}^{3}$, then one can assign $B^{\mathcal{Z}}(X)=\beta(X) \quad \forall X \in \mathcal{Z}$. If $\beta$ is only defined on the nodes of a mesh describing the zero level set of $u$, then one can assign $B^{\mathcal{Z}}(X)=\beta\left(\nu_{i}\right) \quad \forall X \in \mathcal{Z}$, where $\nu_{i}$ is the closest node of the mesh to the voxel $X$. In both cases, the final value of $B(X)$ is not the value of $B^{\mathcal{Z}}(X)$, as explained in the second step.

Notice that the definition of $\mathcal{Z}$ ensures that if $u_{l}(x)=0$ then $L(x) \subset \mathcal{Z}$ and in consequence $B_{l}^{\mathcal{Z}}(x)$ can be computed.

\subsection{Second step: computation of $B$ on the narrow band}

The purpose is to propagate the values of $B$ from $\mathcal{Z}$ to the whole narrow band. This is done by $B(X, t)=B_{l}^{\mathcal{Z}}(y, t)$ where $u_{l}(y)=0$ and $y$ lies on the same characteristic of $u$ than $X$. Computing directly $y=X-u \nabla u$ is not robust since small errors in $\nabla u$ may introduce larger errors (proportional to $u$ ) in $y$. Instead, we follow the characteristic passing through $X$ by unit steps (cf. fig. $(5))$ :

$$
\left\{\begin{aligned}
y_{0} & =X \\
y_{n+1} & =y_{n}-\left\{\begin{array}{ll}
\text { if } & u_{l}\left(y_{n}\right)<0 \\
\text { if } & u_{l}\left(y_{n}\right)>0
\end{array} \text { then } \max \left(u_{l}\left(y_{n}\right), \operatorname{sign}\left(u_{l}\left(y_{n}\right)\right)\right) \nabla_{l} u\left(y_{n}\right)\right. \\
u_{l}\left(y_{n}\right) & =0
\end{aligned}\right.
$$

This marching is done for each voxel in the narrow band, even those of $\mathcal{Z}$. The computation of the march direction $\nabla_{l} u\left(y_{n}\right)$ requires the evaluation of $\nabla u$ at voxels of the grid. The choice of 


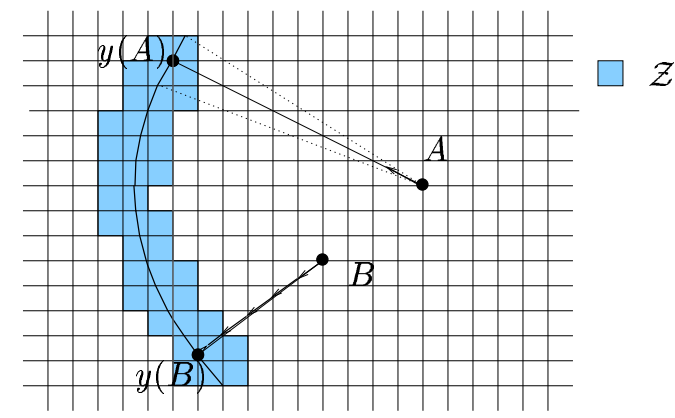

Figure 5: The computation of $y(A)$ by $y(A)=A-u(A) \nabla u(A)$ is potentially subject to large errors. For $B$, the characteristic line is followed by unit steps in order to avoid this error.

the numerical scheme for $\nabla u(X)$ is crucial since it may introduce unrecoverable errors if $X$ lies on the skeleton of $\mathcal{S}$. Our choice is based on the schemes used in the resolution of Hamilton-Jacobi equations where shocks occur $[25,23]$. These schemes use switch functions which turn on or off whenever a shock is detected. We explicit here our choice. Let $D_{x}^{+} u=u(i+1, j, k)-u(i, j, k)$ and $D_{x}^{-} u=u(i, j, k)-u(i-1, j, k)$, with similar expressions for $D_{y}$ and $D_{z}$. We form the eight estimators $D^{i}, i=1, \ldots, 8$ of $\nabla u$, namely:

$$
\begin{aligned}
D^{1} u & =\left(D_{x}^{+} u, D_{y}^{+} u, D_{z}^{+} u\right) \\
D^{2} u & =\left(D_{x}^{+} u, D_{y}^{+} u, D_{z}^{-} u\right) \\
\cdots & \\
D^{8} u & =\left(D_{x}^{-} u, D_{y}^{-} u, D_{z}^{-} u\right)
\end{aligned}
$$

In our current implementation we use $\nabla u(X)=\operatorname{ArgMax}_{i}\left(\left|D^{i} u(X)\right|\right)$. Indeed, apart from points on the skeleton of $\mathcal{S}$ where $\nabla u$ is undefined, $|\nabla u(X)|$ which should be equal to 1 since $u$ is a distance function is found to be in practice less than or equal to 1 depending on which of the operators $D^{i}$ we use. Hence the direction of maximum slope at $X$ is the direction of the closest point to $X$ of the zero level set of $u$. The fact that the skeleton can be detected by comparing the vectors $D^{1} u, D^{2} u, \ldots, D^{8} u$ is discussed in section 6.2 .

\section{Applications}

We now describe three applications where our new method is shown to work significantly better than previous ones.

\subsection{Cortex segmentation using coupled surfaces.}

We have implemented the segmentation of the cortical gray matter (a volumetric layer of variable thickness $(\approx 3 \mathrm{~mm})$ ) from MRI volumetric data using two coupled surfaces proposed in [26] by Zeng et al. The idea put forward in [26] is to evolve simultaneously two surfaces with equations of the form (1). An inner surface $\mathcal{S}_{i n}$ captures the boundary between the white and the gray matter and an outer surface $\mathcal{S}_{\text {out }}$ captures the exterior boundary of the gray matter. The segmented cortical gray matter is the volume between these two surfaces. The velocities of the two surfaces are:

$$
\begin{aligned}
\beta_{\text {in }} & =f\left(I-I_{\text {in }}\right)+C\left(u_{\text {out }}+\epsilon\right) \\
\beta_{\text {out }} & =f\left(I-I_{\text {out }}\right)+C\left(u_{\text {in }}-\epsilon\right)
\end{aligned}
$$

where $I$ is the local gray intensity of the MRI image, $I_{\text {in }}$ and $I_{\text {out }}$ are two thresholds ( $I_{\text {in }}$ for the white matter and $I_{\text {out }}$ for the gray matter), $\epsilon$ is the desired thickness and $C$ and $f$ have the shape of figure (6).

Let us interpret equation (11). The first term $f\left(I-I_{i n}\right)$ forces the gray level values to be close to $I_{\text {in }}$ on $\mathcal{S}_{i n}$ : it is the data attachment velocity term. The second term $C\left(+u_{\text {out }}+\epsilon\right)$ models the 

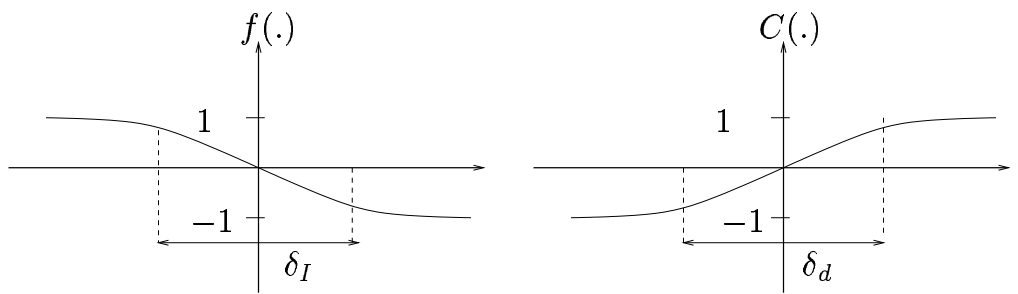

Figure 6: Shapes of the functions $f$ and $C$ in equations $(11,12) . \delta_{I}$ and $\delta_{d}$ are two fixed tolerances.

interaction between $\mathcal{S}_{\text {out }}$ and $\mathcal{S}_{\text {in }}$ : it is the coupling term. According to the shape of $C$, see figure (6), if locally the two surfaces are at a distance $\epsilon=3 \mathrm{~mm}$, then the coupling term has no effect $(C=0)$ and $\mathcal{S}_{\text {in }}$ evolves in order to satisfy its data attachment term. If the local distance between $\mathcal{S}_{\text {in }}$ and $\mathcal{S}_{\text {out }}$ is too small $(<\epsilon)$ then $C>0$ and $\mathcal{S}_{\text {in }}$ slows down in order to get further from $\mathcal{S}_{\text {out }}$. If the local distance between $\mathcal{S}_{i n}$ and $\mathcal{S}_{\text {out }}$ is too large $(>\epsilon)$ then $C<0$ and $\mathcal{S}_{i n}$ speeds up in order to move closer to $\mathcal{S}_{\text {out }}$. A similar interpretation can be done for (12).

If these evolutions are implemented with the Hamilton-Jacobi equation (3), then the following occurs: the magnitudes of the gradients of $u_{\text {out }}$ and $u_{\text {in }}$ increase with time $\left(\left|\nabla u_{\text {out }}\right|>1\right.$ and $\left.\left|\nabla u_{i n}\right|>1\right)$. As a consequence, the estimation of the distance between $\mathcal{S}_{\text {in }}$ and $\mathcal{S}_{\text {out }}$ which is taken as $u_{i n}(x)$ for $x$ on $\mathcal{S}_{\text {out }}$ and $u_{\text {out }}(x)$ for $x$ on $\mathcal{S}_{\text {in }}$, is overestimated. Since the coupling term is negative in (11) and positive in (12), both $\mathcal{S}_{\text {out }}$ and $\mathcal{S}_{\text {in }}$ evolve in order to become closer and closer from each other (until the inevitable reinitialization of the distance functions is performed). In other words, with the standard implementation of the level sets, the incorrect evaluation of the distance functions prevents the coupling term to act correctly and, consequently, also prevents the data attachment terms to play their roles.

On the other hand, if these evolutions are implemented with our new PDE, then a much better interaction between the two terms is achieved since the data attachment term can fully play its role as soon as the distance between the two surfaces is correct (cf. fig. (7)).
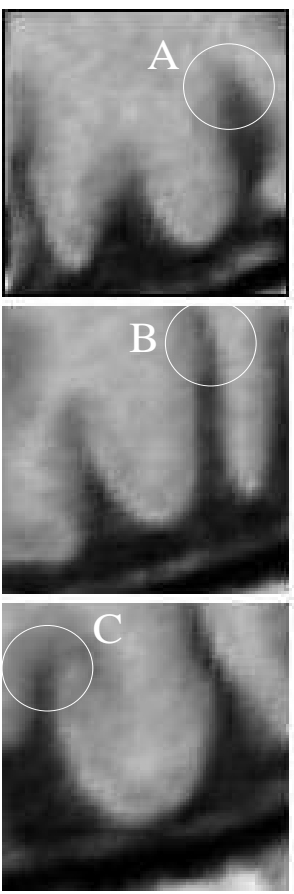
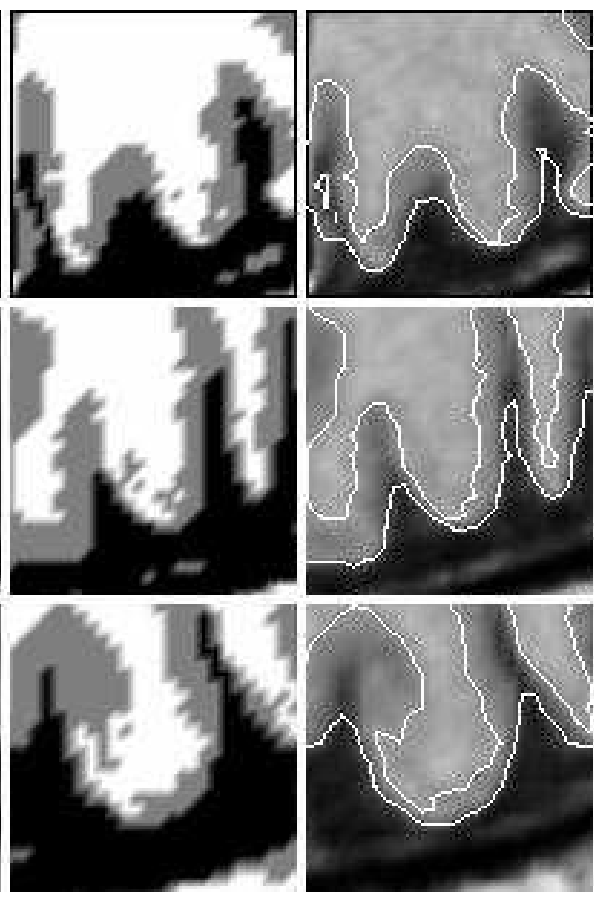
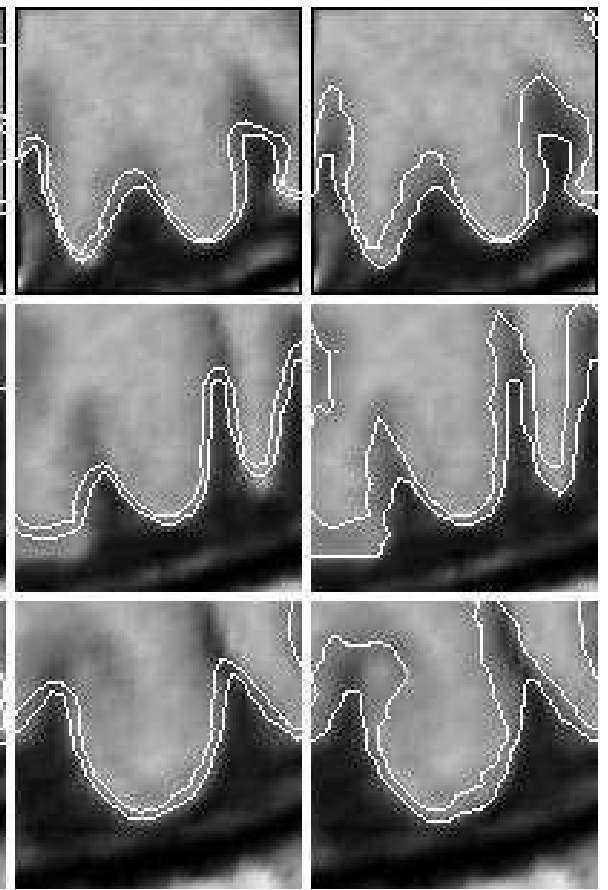

Figure 7: Results of the segmentation of the gray matter using different algorithms, see text.

These results are demonstrated in the figure (7) which we now comment. Each row corresponds to a different $32 \times 32$ sub-slice of an MRI image. The first column shows the original data and some regions of interest (concavities) are labeled A, B and C. The second column shows a simple 
thresholding at $I_{\text {in }}$ and $I_{\text {out }}$. The third column shows the cross-sections of $\mathcal{S}_{\text {in }}$ and $\mathcal{S}_{\text {out }}$ through the slices if the coupling terms are not taken into account. This is why these curves have the same shape as in the second column. One observes that the segmented gray matter has not the wanted regular thickness. In the fourth column, the coupling terms are taken into account and the evolutions (11) and (12) are implemented with Hamilton-Jacobi equation (3). One observes (in particular at the concavities indicated in the first column) that the distance constraint is well satisfied but the data attachment term was neglected. This is due to the fact that with (3) the distance between the two surfaces is overevaluated. In the fifth column, this same evolution is implemented with the new PDE introduced in this paper (10). One can observe a much better result at concavities. This is due to the fact that the coupling terms stop having any effect as soon as the distance between the surfaces is correct allowing the data term to drive correctly the surfaces according to the gray level values. 


\subsection{Extraction of the skeleton of an evolving surface.}

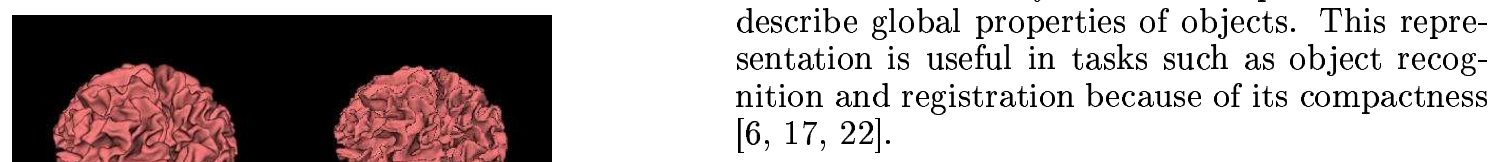

One of the advantages of our new level set technique is that it provides, almost for free, at each time instant a description of the skeleton of the evolving surface or zero level set.

We show an example of this on one of the results of the segmentation described in the previous section. We take the outside surface of the cortex and simplify it using mean-curvature flow, i.e. the evolution $\frac{\partial \mathcal{S}}{\partial t}=H \mathcal{N}$ where $H$ is the mean curvature. This evolution is shown in the first column of figure 8. Since the distance function $u$ to the zero level set is preserved at every step, it is quite simple to extract from it the skeleton by using the fact that it is the set of points where $\nabla u$ is not defined [6]. This is shown in the right column of figure 8. Each surface is rescaled in order to occupy the whole image.

The skeletons are computed using the distance function to the evolving surface as follows. We look for the voxels where the eight estimators $D^{i} u$ of $\nabla u$ defined in section 5 differ a lot and threshold the simple criterion:

$$
\sum_{i}\left(\frac{D^{i} u}{\left|D^{i} u\right|}, \frac{\overline{D u}}{|\overline{D u}|}\right)^{2}
$$

where $(.,$.$) denotes the dot product of two vec-$ tors and $\overline{D u}=\frac{1}{8} \sum_{i} D^{i} u$.

This can be interpreted as a measure of the variations of the direction of $\nabla u$ (which are large in the neighborhood of the skeleton).

The results for the left column of figure (8) are shown in the right column of the same figure where we clearly see how the simplification of the shape of the cortex (left column) goes together with the the simplification of its skeleton (right column).

Note that because it preserves the distance function, our framework allows the use of more sophisticated criteria for determining the skeleton [18] based on this distance function.

Figure 8: Computation of the skeletons of a family of surfaces, see text. 


\subsection{Stereo reconstruction via level sets.}

In this last application, we show how our approach allows a faster convergence when solving the problem of stereo reconstruction from $n \geq 2$ views by means of a PDE-driven surface introduced by Faugeras and Keriven in [12]. More generally, the method described in this article offers significant savings each time the cost of the computation of the velocity term $\beta$ is high. In the stereo application this velocity is given by:

$$
\beta=\phi H-\nabla \phi \cdot \mathcal{N}
$$

where $H$ is the mean curvature of $\mathcal{S}$ and $\phi$ is a measure of the local similarity of two of the $n$ images of the tridimensional scene to be reconstructed. Let us qualitatively compare the cost (in time) of implementing stereo, equation (13), to the cost of implementing mean curvature flow for which $\beta=H$.

$\phi$ is derived from the normalized cross-correlation of two small sub-images (say of size $n=$ $15 \times 15$ ). The number of multiplications (the most costly operation) is $3 n$. Indeed, let $a$ and $b$ be two vectors of length $n$. The calculation of their normalized cross correlation [11] mainly requires the calculation of the three dot products $(a, a),(b, b)$ and $(a, b)$ (i.e., for computing this criterion for two images of size $15 \times 15$, one reorders the pixels values in a vector of length $n=15 \times 15$, which shows that 675 multiplications are needed in this case). Computing $H$ requires 25 multiplications. As a consequence, one iteration of (13) is approximatively 30 times slower than one iteration of the mean curvature flow. This is the main reason why the convergence of the stereo algorithm (13) is slow (about $2 \mathrm{~h} 30$ on a Sun30 with $n=3$ images and a $100^{3}$ grid) and why it is important to speed it up.

Notice however that in our approach $\beta$ is evaluated much fewer times (Indeed, $\beta$ is evaluated only on $\mathcal{Z}$ and not on the whole narrow band). Moreover, the second step (section 5.2) of our algorithm is independent of the specific application: this is why our method is so advantageous in applications where the computation of $\beta$ is very expensive. (9).

The following table shows the considerable gain obtained in the experiment described in figure

\begin{tabular}{|c|c|c|c|}
\cline { 2 - 4 } \multicolumn{1}{c|}{} & \multicolumn{3}{c|}{ timings of the reconstruction on a Sun30 } \\
\hline band half width & $\frac{\partial u}{\partial t}=\beta|\nabla u|$ & $\frac{\partial u}{\partial t}=\beta(x-u \nabla u)$ & gain \\
\hline 4 & $8856 s$ & $6730 s$ & $24 \%$ \\
\hline 8 & $19748 s$ & $10998 s$ & $44 \%$ \\
\hline
\end{tabular}

\section{Conclusion}

We have proposed a new scheme for solving the problem of evolving through the technique of level sets a surface $\mathcal{S}(t)$ satisfying a PDE such as (1). This scheme introduces a new PDE, (10), that must be satisfied by the auxiliary function $u(t)$ whose zero level set is the surface $\mathcal{S}(t)$. The prominent feature of the new scheme is that the solution to this PDE is the distance function to $\mathcal{S}(t)$ at each time instant $t$. Our approach has many theoretical and practical advantages that were discussed and demonstrated on three applications. Since the distance function to the evolving surface is in most applications the preferred function, we believe that the PDE that was presented here is an interesting alternative to Hamilton-Jacobi equations which do not preserve this function. 


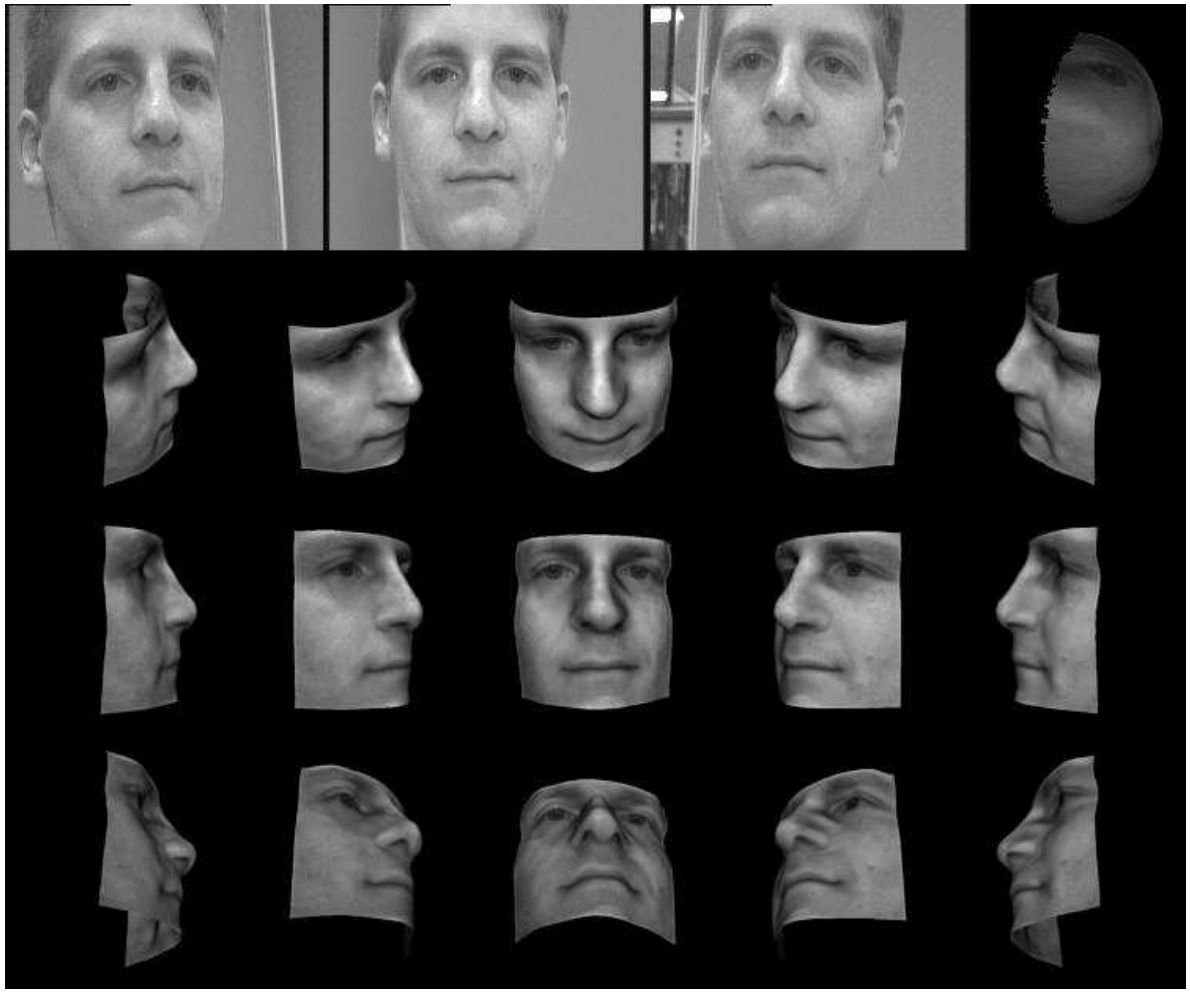

Figure 9: The three images on the top left were taken simultaneously from different points of view. The image on the top right shows the initial surface (a sphere) with the three images back-projected on it. The reconstruction was obtained by deforming this sphere according to (1) with $\beta$ given by (13). The remaining 15 images show the resulting reconstruction from various points of view.

\section{References}

[1] D. Adalsteinsson and J. A. Sethian. A Fast Level Set Method for Propagating Interfaces. Journal of Computational Physics, 118(2):269-277, 1995.

[2] D. Adalsteinsson and J. A. Sethian. The fast construction of extension velocities in level set methods. Technical Report PAM-738, Center for Pure and Applied Mathematics. University of California at Berkeley, 1997.

[3] L. Ambrosio and C. Mantegazza. Curvature and distance function from a manifold. J. Geom. Anal., 1996. To appear.

[4] V. I. Arnold. Geometrical Methods in the Theory of Ordinary Differential Equations. SpringerVerlag New York Inc., 1983.

[5] G. Barles, H.M. Soner, and P.E. Souganidis. Front propagation and phase field theory. SIAM J. Control and Optimization, 31(2):439-469, March 1993.

[6] Harry Blum and Roger N. Nagel. Shape description using weighted symmetric axis features. Pattern Recog., 10:167-180, 1978.

[7] V. Caselles, R. Kimmel, and G. Sapiro. Geodesic active contours. In Proceedings of the 5th International Conference on Computer Vision, pages 694-699, Boston, MA, June 1995. IEEE Computer Society Press.

[8] Vicent Caselles, Ron Kimmel, and Guillermo Sapiro. Geodesic active contours. The International Journal of Computer Vision, 22(1):61-79, 1997.

[9] Y.G. Chen, Y. Giga, and S. Goto. Uniqueness and existence of viscosity solutions of generalized mean curvature flow equations. J. Differential Geometry, 33:749-786, 1991. 
[10] David L. Chopp. Computing minimal surfaces via level set curvature flow. Journal of Computational Physics, 106:77-91, 1993.

[11] Frédéric Devernay. Vision stéréoscopique et propriétés différentielles des surfaces. $\mathrm{PhD}$ thesis, École Polytechnique, February 97.

[12] O. Faugeras and R. Keriven. Level set methods and the stereo problem. In Bart ter Haar Romeny, Luc Florack, Jan Koenderink, and Max Viergever, editors, Proc. of First International Conference on Scale-Space Theory in Computer Vision, volume 1252 of Lecture Notes in Computer Science, pages 272-283. Springer, 1997.

[13] M. Gage and R.S. Hamilton. The heat equation shrinking convex plane curves. J. of Differential Geometry, 23:69-96, 1986.

[14] M. Grayson. The heat equation shrinks embedded plane curves to round points. J. of Differential Geometry, 26:285-314, 1987.

[15] M. Kass, A. Witkin, and D. Terzopoulos. SNAKES: Active contour models. The International Journal of Computer Vision, 1:321-332, January 1988.

[16] S. Kichenassamy, A. Kumar, P. Olver, A. Tannenbaum, and A. Yezzi. Gradient flows and geometric active contour models. In Proc. Fifth International Conference on Computer Vision, Boston, MA, June 1995. IEEE Computer Society Press.

[17] B. Kimia, A. R. Tannenbaum, and S. W. Zucker. Shapes, schoks and deformations i: The components of two-dimensional shape and the reaction-diffusion space. ijcv, 15:189-224, 1995.

[18] G. Malandain and S. Fernández-Vidal. Euclidean skeletons. Image and Vision Computing, 16:317-327, 1998.

[19] R. Malladi, J. A. Sethian, and B.C. Vemuri. Evolutionary fronts for topology-independent shape modeling and recovery. In J-O. Eklundh, editor, Proceedings of the 3rd European Conference on Computer Vision, volume 800 of Lecture Notes in Computer Science, Stockholm, Sweden, May 1994. Springer-Verlag.

[20] R. Malladi, J. A. Sethian, and B.C. Vemuri. Shape modeling with front propagation: A level set approach. PAMI, 17(2):158-175, February 1995.

[21] S. Osher and J. Sethian. Fronts propagating with curvature dependent speed : algorithms based on the Hamilton-Jacobi formulation. Journal of Computational Physics, 79:12-49, 1988.

[22] Jean Serra. Image Analysis and Mathematical Morphology. Academic Press, London, 1982.

[23] J. A. Sethian. Level Set Methods. Cambridge University Press, 1996.

[24] J.A. Sethian and J. Strain. Crystal growth and dendritic solidification. Journal of Computational Physics, 98:231-253, 1992.

[25] C. W. Shu and S. Osher. Efficient implementation of essentially non-oscillatory shockcapturing schemes, ii. Journal of Computational Physics, 83:32-78, 1989.

[26] X. Zeng, L. H. Staib, R. T. Schultz, and J. S. Duncan. Volumetric layer segmentation using coupled surfaces propagation. In Proceedings of the International Conference on Computer Vision and Pattern Recognition, Santa Barbara, 1998. 


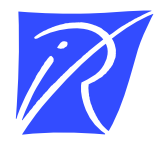

Unité de recherche INRIA Sophia Antipolis

2004, route des Lucioles - B.P. 93 - 06902 Sophia Antipolis Cedex (France)

Unité de recherche INRIA Lorraine : Technopôle de Nancy-Brabois - Campus scientifique 615, rue du Jardin Botanique - B.P. 101 - 54602 Villers lès Nancy Cedex (France)

Unité de recherche INRIA Rennes : IRISA, Campus universitaire de Beaulieu - 35042 Rennes Cedex (France)

Unité de recherche INRIA Rhône-Alpes : 655, avenue de l'Europe - 38330 Montbonnot St Martin (France)

Unité de recherche INRIA Rocquencourt : Domaine de Voluceau - Rocquencourt - B.P. 105 - 78153 Le Chesnay Cedex (France)

Éditeur

INRIA - Domaine de Voluceau - Rocquencourt, B.P. 105 - 78153 Le Chesnay Cedex (France)

http://www.inria.fr

ISSN 0249-6399 